\title{
Simulation of Microgravity by Magnetic Levitation and Random Positioning: Effect on Human A431 Cell Morphology
}

\author{
Maarten J. A. Moes • Jeroen C. Gielen • \\ Robert-Jan Bleichrodt • Jack J. W. A. van Loon • \\ Peter C. M. Christianen • Johannes Boonstra
}

Received: 24 August 2009 / Accepted: 25 February 2010 / Published online: 17 March 2010

(C) The Author(s) 2010. This article is published with open access at Springerlink.com

\begin{abstract}
Simulation of weightlessness is a desired replenishment for research in microgravity since access to space flights is limited. In real microgravity conditions, the human epidermoid cell line A431 exhibits specific changes in the actin cytoskeleton resulting ultimately in the rounding-up of cells. This rounding of A431 cells was studied in detail during exposure to Random Positioning Machine (RPM) rotation and magnetic levitation. Random rotation and magnetic levitation induced similar changes in the actin morphology of A431 cells that were also described in real microgravity. A transient process of cell rounding and renewed spreading was observed in time, illustrated by a changing actin cytoskeleton and variation in the presence of focal adhesions. However, side effects of both methods easily can lead to false linking of cellular responses to simulated microgravity. Therefore further characterization of both methods is required.
\end{abstract}

M. J. A. Moes · R. Bleichrodt · J. Boonstra ( $\varangle)$ Cellular Architecture and Dynamics,

Institute of Biomembranes, Utrecht University, Padualaan 8, 3584 CH Utrecht, The Netherlands

e-mail: J.Boonstra@uu.nl

J. C. Gielen · P. C. M. Christianen

High Field Magnet Laboratory, Institute for Molecules and Materials, Radboud University Nijmegen, Toernooiveld 7, 6525 ED Nijmegen, The Netherlands

J. J. W. A. van Loon

Dutch Experiment Support Center (DESC),

Department of Oral Cell Biology, ACTA,

University of Amsterdam-Vrije Universiteit,

Amsterdam, The Netherlands
Keywords Actin - Magnetic levitation - RPM • Simulated microgravity • Weightlessness • Focal adhesion $\cdot$ FAK
Abbreviations
RPM random positioning machine
FAK focal adhesion kinase
EGF epidermal growth factor

\section{Introduction}

During the last decades a wide variety of space flight experiments have demonstrated that gravity has profound effects on whole organisms, organs and tissues, resulting for example in bone and muscle mass reduction as well as in the occurrence of cardiovascular malfunctioning and many other processes (Carmeliet et al. 2001; Fitts et al. 2001; Fritsch-Yelle et al. 1996). Interestingly, the virtual absence of gravity also had profound effects on the cellular and molecular level, including changes in cell morphology (Rijken et al. 1991a; Hughes-Fulford et al. 2003), modification of gene expression (de Groot et al. 1991a; Hammond et al. 1999; Liu and Wang 2008), changes in signal transduction cascades (de Groot et al. 1991b; Ullrich et al. 2008) and even changes in the selforganisation of tubulin (Papaseit et al. 2000; Glade et al. 2006; Tabony et al. 2007).

Thus it was demonstrated that exposure of epidermoid A431 cells to microgravity conditions during a sounding rocket flight, resulted in a significant decrease of EGF-induced $c$-fos and $c$-jun expression (de Groot et al. 1990). Subsequent experiments demonstrated that the effect of microgravity on gene expression was a 
specific effect, as for example the EGF and phorbol ester-induced $c$-fos expression were sensitive to microgravity, but the forskolin and A23187-induced c-fos were insensitive, suggesting that in particular the protein kinase $\mathrm{C}$ (PKC)-mediated signal transduction cascades were sensitive to gravity (de Groot et al. 1991b). In addition it was demonstrated during sounding rocket flights that microgravity exposure of A431 cells resulted in increased actin polymerization and cell rounding (Rijken et al. 1991a), a process that is dependent on actin. Since PKC activation is dependent upon actin polymerization, these findings suggested that the actin microfilament system may represent a gravity sensitive component in cells (Boonstra 1999).

Actin is a major component of the cytoskeleton and has important functions, amongst others in signal transduction, motility, attachment, and cell morphology, (for review see Boonstra and Moes 2005). Actin is present both in a non-polymerized form (G-actin) and a polymerized form (F-actin). Polymerized actin can form various structures in cells such as stress fibers and the cortical skeleton that together determine the morphology of cells. The state of actin is tightly regulated by actin binding proteins (ABPs) as described in detail in several recent review papers (dos Remedios et al. 2003; Boonstra and Moes 2005).

Microgravity based research is hampered by a very limited access to space flights, both short lasting conditions (sounding rockets) and long duration missions (Space Shuttle, or International Space Station, ISS), making routine laboratory research impossible. Therefore attempts have been made to develop conditions in which microgravity was simulated. One of the first successful approaches for single cell studies concerns the fast rotating clinostat, a device that enables the rotation of a cell culture around an axis perpendicular to the gravity vector (Briegleb 1992). Thus it was demonstrated that both the reduction in gene expression and the increase in actin polymerization, as measured during sounding rocket experiments, were also observed under simulated microgravity using a fast rotating clinostat (Rijken et al. 1991a). Improvements of the principles of the fast rotating clinostat resulted in the development of the random positioning machine (RPM; Hoson et al. 1992, 1997; van Loon 2007). In the RPM, samples are mounted on a platform that randomly changes position in three dimensions by driving two independent frames that rotate independently in random directions and at random speeds. The random rotation in all directions results in a net force of zero. Simulation of weightlessness with the RPM was reported to result in a wide variety of cellular responses which have also been found partly under real micro- gravity conditions (Hoson et al. 1997; Schwarzenberg et al. 1998; Grimm et al. 2002; Uva et al. 2002; Infanger et al. 2006; Ulbrich et al. 2008; Versari et al. 2007a, b).

Both the fast rotating clinostat and the RPM are based on the principle that the direction of gravity is randomized. This randomization requires time and therefore only processes can be studied that experience a certain time lag phase. Since the randomization time is dependent on the rotation speed, the processes studied may require also different rotation speeds, dependent on their intrinsic time lag phase. For this reason the clinostat and RPM are not suitable for relatively fast occurring molecular and cellular processes. Perception of residual levels of gravity will depend on the time lag phase, speed of rotation and position of the sample within the system. Increasing the speed of rotation will lower residual gravity perception. On the other hand, increasing the speed of rotation might increase cellular responses introduced by other parameters, such as centrifugal forces.

An alternative to this end may be provided by magnetic levitation (Beaugnon and Tournier 1991a, b). In this technique magnetic forces are exerted on diamagnetic objects by positioning them in a strong gradient magnetic field. Since diamagnetic objects are repelled by magnetic fields this results in a magnetic force, towards regions of low field strength, that can be used to counterbalance the gravitational force, resulting in stable levitation and the simulation of microgravity. Since the vast majority of materials is diamagnetic, it is possible to magnetically levitate a wide variety of substances, such as water, organic solvents and Bismuth (Beaugnon and Tournier 1991a, b). Surprisingly, also more complex biological objects can be levitated, including frog eggs, cells and living frogs and grasshoppers (Berry and Geim 1997; Valles et al. 1997; Geim 1998; Glade et al. 2006), despite the fact that they consist of components with slightly different magnetic susceptibility. Because the gravitational force is compensated on the level of individual molecules, the condition for magnetic levitation depends on the quotient of the magnetic susceptibility of a material and its density. This quotient is remarkably constant for the typical constituents of biological systems, such as water, blood, tissues and bones, with mutual deviations of only a few percent (Schenck 1992). Complex biological systems are therefore relatively homogeneous with respect to diamagnetic levitation. Additional advantages of magnetic levitation are the tuneability of the effective gravity by varying the applied magnetic field strength (Heijna et al. 2007) and the fact that it provides simulated microgravity almost instantly, allowing the study of relatively fast processes. 
The aim of the present research was to use random positioning and magnetic levitation to study the effect of these microgravity simulation paradigms on the actin cytoskeleton in human A431 cells. We compare the results with data found in the past in real microgravity and in simulated microgravity using the fast rotating clinostat. However, during magnetic levitation cells are exposed to high magnetic fields. Therefore we studied also the effect of such a magnetic field on the cells without levitation.

Human A431 cells were exposed to random positioning and magnetic levitation for different time intervals and chemically fixed while rotation or levitation was ongoing. Subsequently the actin morphology and behaviour of focal adhesions were investigated using fluorescence microscopy. The presence of focal adhesions is an indicator for attachment and rounding or spreading of cells. Interestingly, identical results were obtained in the RPM studies and magnetic levitation studies. However, controls for the effect of the magnetic field raised concern about the potential of magnetically simulated microgravity to measure the effects of microgravity on cell morphology and also indicated the importance of this control. Also simulation with the RPM raised concerns about side-effects. Though with both methods results obtained in real microgravity were repeated, the further characterization of both methods is required.

\section{Materials and Methods}

\section{Materials}

Tissue culture nutrients, $\mathrm{CO}_{2}$ independent Dulbecco's modified Eagle medium (DMEM) and fetal bovine serum (FBS) were purchased from Gibco/Invitrogen (Paisley, UK). All other chemicals used were obtained from Sigma-Aldrich (St. Louis, USA) or Merck (Darmstadt, Germany) and were of the highest purity available. The anti-phospho-FAK ${ }^{397}$ and anti-phosphoFAK $^{576}$ antibodies were purchased from Biosource/ Invitrogen (Paisley, UK). The Alexa 488 conjugated anti rabbit antibody was obtained from Molecular Probes/ Invitrogen (Paisley, UK).

\section{Cell Culture}

Human A431 cells were grown at $37^{\circ} \mathrm{C}$ in $\mathrm{CO}_{2}$ independent medium supplemented with $7.5 \%$ FBS and $5 \mathrm{mM}$ L-glutamine. Cells were plated on glass coverslips and cultured for 1 or 2 days at respectively 15.000 or 30.000 cells per $\mathrm{cm}^{2}$. Cells used for EGF stimulation
(80 $\mathrm{ng} / \mathrm{ml}$ EGF) and RPM experiments were serum starved for $24 \mathrm{~h}$.

\section{Random Positioning Machine}

The random positioning machine was manufactured by Dutch Space B.V. (Leiden, The Netherlands). The RPM consists of an inner and an outer frame that can rotate independently in random directions and random speeds. The inner frame rotates within the outer frame. For RPM modes; random direction, random interval and random speed was set and the maximum random speed was chosen as $360^{\circ} / \mathrm{s}$.

In the middle of the inner rotating frame, a device was placed that allows automated refreshment of fluids while the RPM is rotating. This COmpact BioReactor Assembly or COBRA (Dutch Space, Leiden, NL; Borst and van Loon 2009) can hold a 12 well plate as a sample chamber. One well of a 12 wells plate was used and placed in the center of the rotating axes. A coverslip with cells was fixed to the bottom of this well and the well was subsequently filled with medium without gas bubbles. Cells were fixed with $4 \%$ formaldehyde at various time intervals while rotation of the RPM was ongoing. The maximum distance of the sample to the center of rotation was less than $1 \mathrm{~cm}$. In combination with a speed of rotation of $360 \%$ s this results in a residual $\mathrm{g}$ between $10^{-3}$ and $10^{-2} \mathrm{~g}$, for $360^{\circ} / \mathrm{s}$ (van Loon 2007). Experiments were performed in a humidified room at $37^{\circ} \mathrm{C}$.

\section{Magnetic Levitation}

For performing experiments in high gradient magnetic fields, a dedicated culture chamber was constructed that holds a coverslip with cells surrounded by culture medium (Fig. 1). An entrance in the chamber allowed washing and fixing, while the cells were exposed to magnetic levitation.

After insertion of the coverslip in the experimental culture chamber, the cells remained untreated for 14 min during which the temperature was brought to $36.9 \pm 0.1^{\circ} \mathrm{C}$. The strength of the magnetic field was reached in approximately $1 \mathrm{~min}$, using a $20 \mathrm{~T}$, watercooled Bitter magnet (Perenboom et al. 2003). The levitation conditions of water were used, for which the magnetic field strength times the field gradient is equal to $1,401 \mathrm{~T}^{2} / \mathrm{m}$. In practice the magnetic field was set to $17 \mathrm{~T}$ in the center of the magnet, resulting in a field strength of $11.5 \mathrm{~T}$ at the position of levitation, $7.2 \mathrm{~cm}$ above the field center. For magnetic field controls the samples were placed in the center of the magnet using a spatially homogeneous field of $11.5 \mathrm{~T}$. The samples 


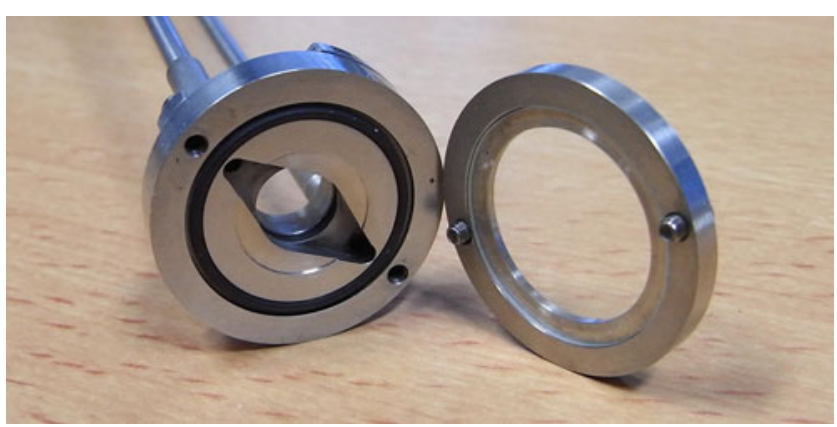

Fig. 1 Opened culture chamber that was used for experiments in the magnet. The central circle provides space for a $12 \mathrm{~mm}$ coverslip. After insertion of a coverslip, a glass lid was placed on top of the chamber. The two tubes at the other side of the chamber were used for fluid refreshment, allowing chemical fixation while the experiment was ongoing

were exposed to the magnetic fields for various time intervals and chemically fixed by flushing the sample chamber with $4 \%$ formaldehyde while levitation was ongoing. For these experiments the duration of levitation was $0,6,30,60$ and $120 \mathrm{~min}$. The data for 6 and $30 \mathrm{~min}$ represent three or four independent experiments, the later time points two or three independent experiments.

\section{Immunofluorescence Labeling}

EGF stimulated samples were fixed for 30 min with $4 \%$ formaldehyde. Chemically fixed samples of magnetic levitation experiments were transported back to the laboratory at room temperature and kept overnight at $4^{\circ} \mathrm{C}$. RPM samples were also kept overnight at $4^{\circ} \mathrm{C}$ before further processing the next day. Samples were washed extensively with PBS to remove formaldehyde, permeabilized for $5 \mathrm{~min}$ in PBS containing $0.2 \%$ Triton X-100, followed by two washes in PBS, and incubated for 10 min with $50 \mathrm{mM}$ glycine in PBS. After washing twice with PBS containing $0.2 \%$ gelatine, cells were incubated for $60 \mathrm{~min}$ at room temperature with the primary antibody raised against phospho-FAK ${ }^{397}$ or phospho-FAK ${ }^{576}$. This was followed by washing six times in PBS containing $0.2 \%$ gelatin. Subsequently, cells were incubated for $60 \mathrm{~min}$ with (TRITC) or FITC-conjugated phalloidin and the GAR Alexa 488 or GARCy3 antibody. Finally, cells were mounted in Mowiol-DABCO.

\section{Acquisition of Images}

Results represented in Fig. 2 were visualized with a Leica microscope (Orthoplan) fitted with a $\times 40$ N.A.
1.3 Leica oil objective. Images were acquired with a Leica CCD camera (model DC350F) using Leica Image Manager 50 software. Results represented in Fig. 3 were visualized with an Olympus microscope (AX70) fitted with a $\times 60$ N.A. 1.25 Olympus Uplan Fl objective. Images were acquired with a Nikon CCD camera (DXM1200) using Nikon ACT1 software. Results represented in Figs. 4 and 5 were visualized with a Zeiss microscope (Axioskop) equipped with a Zeiss objective $\times 40$, N.A. 0.75 oil. This microscope was coupled to a Leica CCD camera (DFC420C) and images were acquired using Leica Image Manager software. Subsequently pictures were processed with Abobe Photoshop 8.0.

\section{Results}

\section{Rounding of A431 Cells}

Under normal, $1 \mathrm{~g}$ conditions human epidermoid carcinoma A431 cells grow in clusters and are not fully flattened but semi rounded. This is indicated by the clear presence of a cortical F-actin skeleton (Fig. 2a). The degree of rounding of cells can be further established by the visualization of the focal adhesions. A cell that is spread exhibits clear focal adhesions whereas a cell that is fully rounded will show little or no focal adhesions.

A431 showed reduced adhesion upon stimulation with epidermal growth factor (EGF; Nelson and Fry 1997; Genersch et al. 1998; Rijken et al. 1991b). This EGF induced modification in adhesion of A431 cells was used as a model to find markers for cell rounding in RPM and magnetic levitation studies. Within 5 min of EGF stimulation $(80 \mathrm{ng} / \mathrm{ml})$ phospho-FAK ${ }^{397}$ labelling disappears in focal adhesions (Fig. 2d). Furthermore the cortical F-actin skeleton becomes more pronounced and stress fibres disappear (Fig. 2c). Cells retract thereby covering less surface area and retraction spikes indicate this process (Fig. $2 \mathrm{~d}$ insert). The rounding of cells is followed by spreading, indicating the transient nature of this process. Thirty minutes after initial stimulation with EGF the cortical skeleton becomes less pronounced, cells show renewed labelling for phospho-FAK ${ }^{397}$ in focal adhesions (Fig. 2f) and newly formed stress fibers (Fig. 2e) indicating a more flattened morphology. Together, the behavior of Factin and phospho-FAK ${ }^{397}$ labelling of focal adhesions, give a clear indication of the degree of rounding of A431 cells. 
Fig. 2 EGF induced cell rounding of A431 cells. A431 cells were serum starved for $24 \mathrm{~h}$ and subsequently incubated in the presence of $80 \mathrm{ng} / \mathrm{ml}$ EGF for 0,5 and $30 \mathrm{~min}$ at $37^{\circ} \mathrm{C}$, respectively $(\mathbf{a}, \mathbf{b}),(\mathbf{c}, \mathbf{d})$ and $(\mathbf{e}, \mathbf{f})$. After fixation, immunofluorescence labelling was performed using Phalloidin-FITC (green) to stain F-actin $(\mathbf{a}, \mathbf{c}, \mathbf{e})$ and anti-phospho-FAK ${ }^{397}$ (red) to stain focal adhesions $(\mathbf{b}, \mathbf{d}, \mathbf{f})$. In non-stimulated cells F-actin is visible both in stress fibers (arrow) and in the cortical skeleton (a). Phospho-FAK ${ }^{397}$ labelling is present in focal adhesions (b) (insert). Five minutes after EGF stimulation the number of stress fibers is reduced and cells are more rounded (c), the number of focal adhesions that are labelled with phospho-FAK ${ }^{397}$ is strongly reduced (d) (insert). Thirty minutes after EGF stimulation cells flatten again indicated by the increase in the number of stress fibers (E, arrow) and the labelling for phospho-FAK ${ }^{397}$ in the focal adhesions (f) (insert). Bar represents $10 \mu \mathrm{m}$, all inserts are enlarged twice
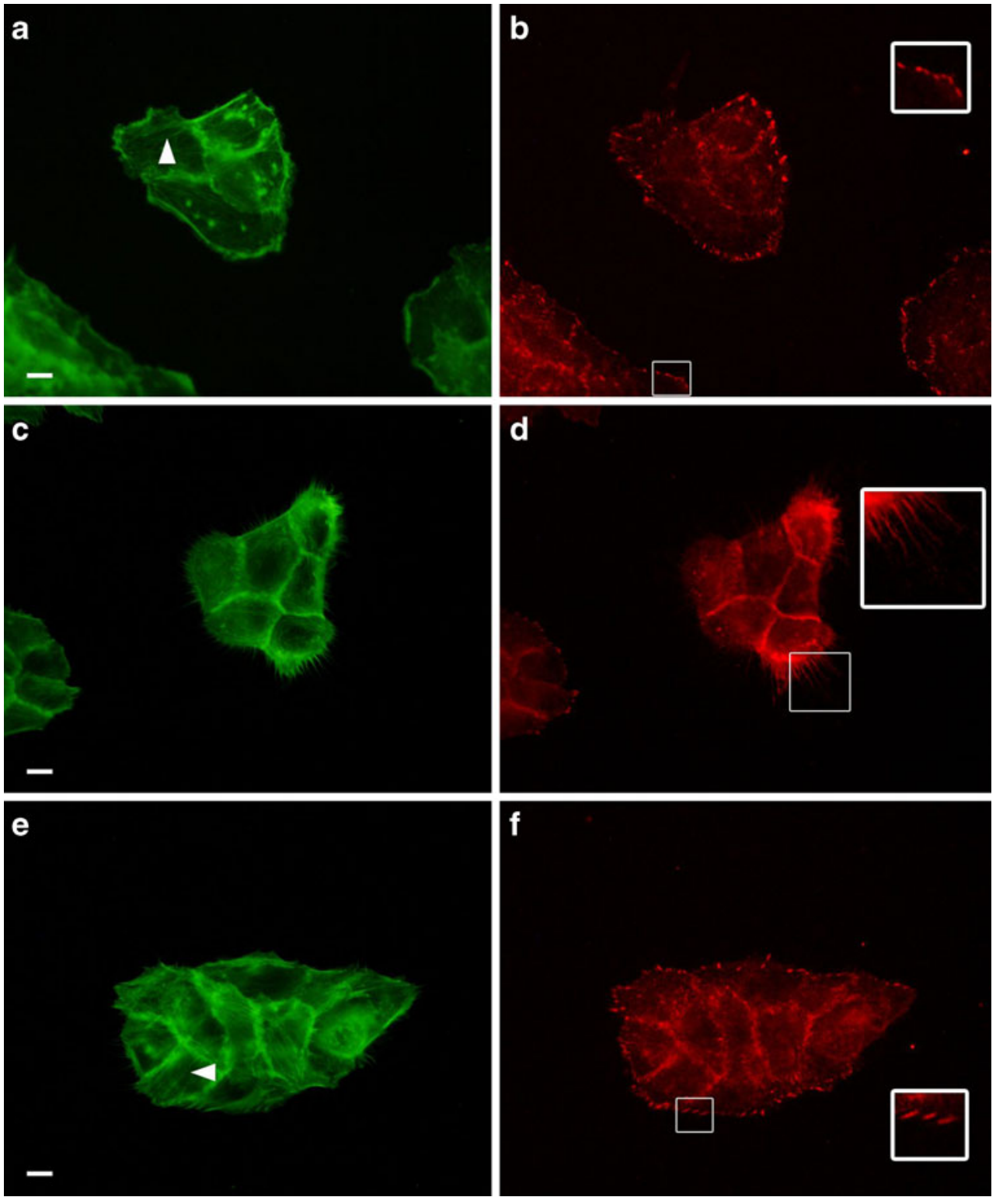

Effect of Simulated Microgravity on A431 Cell Morphology Using the RPM

In order to study the effect of simulated microgravity on A431 cell morphology, the cells were placed in the RPM as described under "Materials and Methods". As shown in Fig. 3c, the cortical actin skeleton became more pronounced after 6 min of simulated microgravity. After $30 \mathrm{~min}$ of rotation the cells showed increased rounding (Fig. 3e), followed by spreading of cells after $60 \mathrm{~min}$ of rotation (Fig. 3g). This pattern of rounding and spreading was supported by the visualization of phospho-FAK ${ }^{576}$ labelling in focal adhesions. The phosphorylation of FAK at pY576 in focal adhesions during cell rounding is comparable with that of pY397 (data not shown). After 6 min of RPM rotation, the number of focal adhesions stained for phospho-FAK ${ }^{576}$ decreased but remaining focal adhesions showed intense labelling for phospho-FAK ${ }^{576}$ (Fig. 3d). After 30 min of rotation (Fig. 3f), the number of focal adhesions stained for phospho-FAK ${ }^{576}$ was clearly further decreased as compared to cells after 0 (Fig. 3b) and $6 \mathrm{~min}$ (Fig. 3d) of rotation while after $60 \mathrm{~min}$ of rotation (Fig. 3h) the number of focal adhesions detected with the anti-phospho-FAK ${ }^{576}$ antibody increased again. This indicates renewed attachment and spreading of cells.

These experiments clearly demonstrate that A431 cell morphology showed a transient process of rounding and subsequent reattachment during rotation in the 
Fig. 3 A431 cells exposed to 3D RPM rotation. Cells were rotated with random speed, direction and interval (Borst and van Loon 2009) for 0, 6, 30 and $60 \mathrm{~min}$, respectively $(\mathbf{a}, \mathbf{b}),(\mathbf{c}, \mathbf{d}),(\mathbf{e}, \mathbf{f})$ and $(\mathbf{g}, \mathbf{h})$. After 6 min cells started to round $(\mathbf{c})$ and the number of focal adhesions labelled with anti-phospho-FAK ${ }^{576}$

decreased (d) but remaining focal adhesions increased in size and labelled for both

F-actin (red) and phospho-FAK (green). Also retraction spikes appeared (arrows in c, d). After $30 \mathrm{~min}$ of rotation the number of detected focal adhesions is further decreased (f) and cells occupied less surface area indicating cell rounding (e). At 60 min cells were spread again (g) and a large number of focal adhesions was stained with anti-phospho-FAK ${ }^{576}$

(h). Bar represents $10 \mu \mathrm{m}$, all inserts are enlarged twice
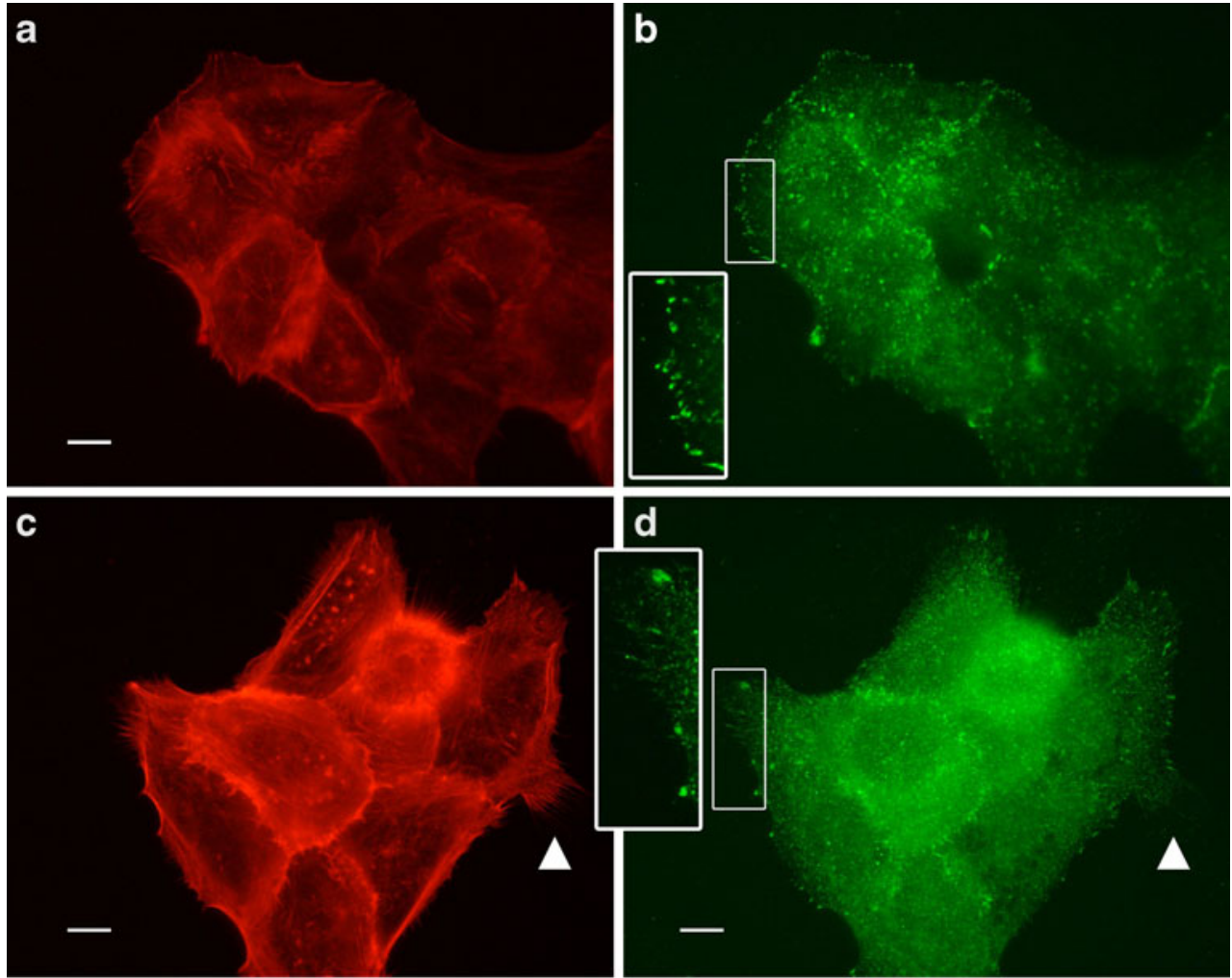

d
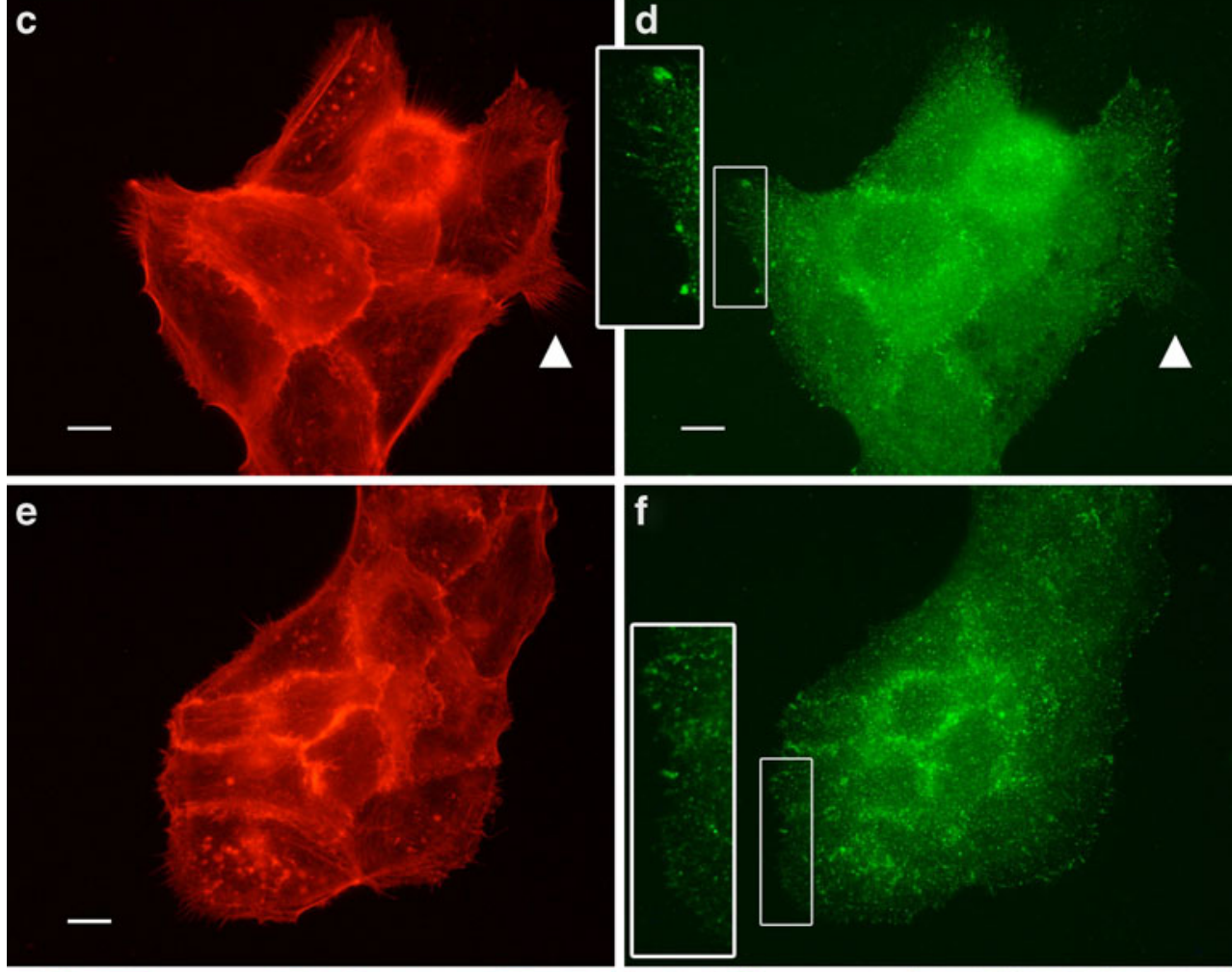

g

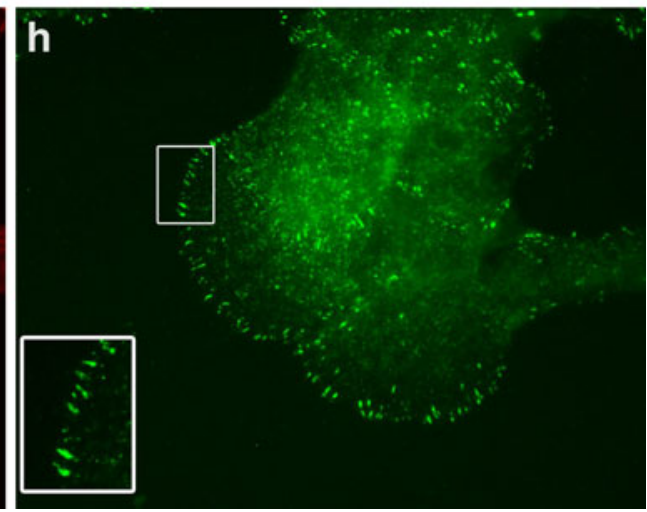

RPM. The rounding-up of cells after 6 min of rotation is comparable to the results obtained in real microgravity using sounding rockets and in the fast rotating clinostat (Rijken et al. 1991a).
Effect of Magnetic Levitation on A431 Cell Morphology

To induce magnetic levitation, cells were subjected to a gradient magnetic field of $1,401 \mathrm{~T}^{2} / \mathrm{m}, 7.2 \mathrm{~cm}$ above 
Fig. 4 Magnetic levitation of A431 cells for $0,6,30,60$ and 120 min. Magnetic levitation results in the rounding and subsequent spreading of cells, indicated by the increased cortical F-actin skeleton (red) and disappearance of phospho-FAK ${ }^{397}$ labelling (green) in focal adhesions during rounding. Only after 6 min of levitation cells are already rounding $(\mathbf{c}, \mathbf{d})$. After $30 \mathrm{~min}(\mathbf{e}, \mathbf{f})$ and $60 \mathrm{~min}(\mathbf{g}, \mathbf{h})$ cells round even more. The cells occupied less surface area, showed no stress fibers, had a pronounced cortical skeleton and no phospho-FAK staining in focal adhesions. Spreading is indicated by the

reappearance of stress fibers (i) and renewed phospho-FAK ${ }^{397}$ labelling in focal adhesions (j). Bar represents $10 \mu \mathrm{m}$, all inserts are enlarged twice
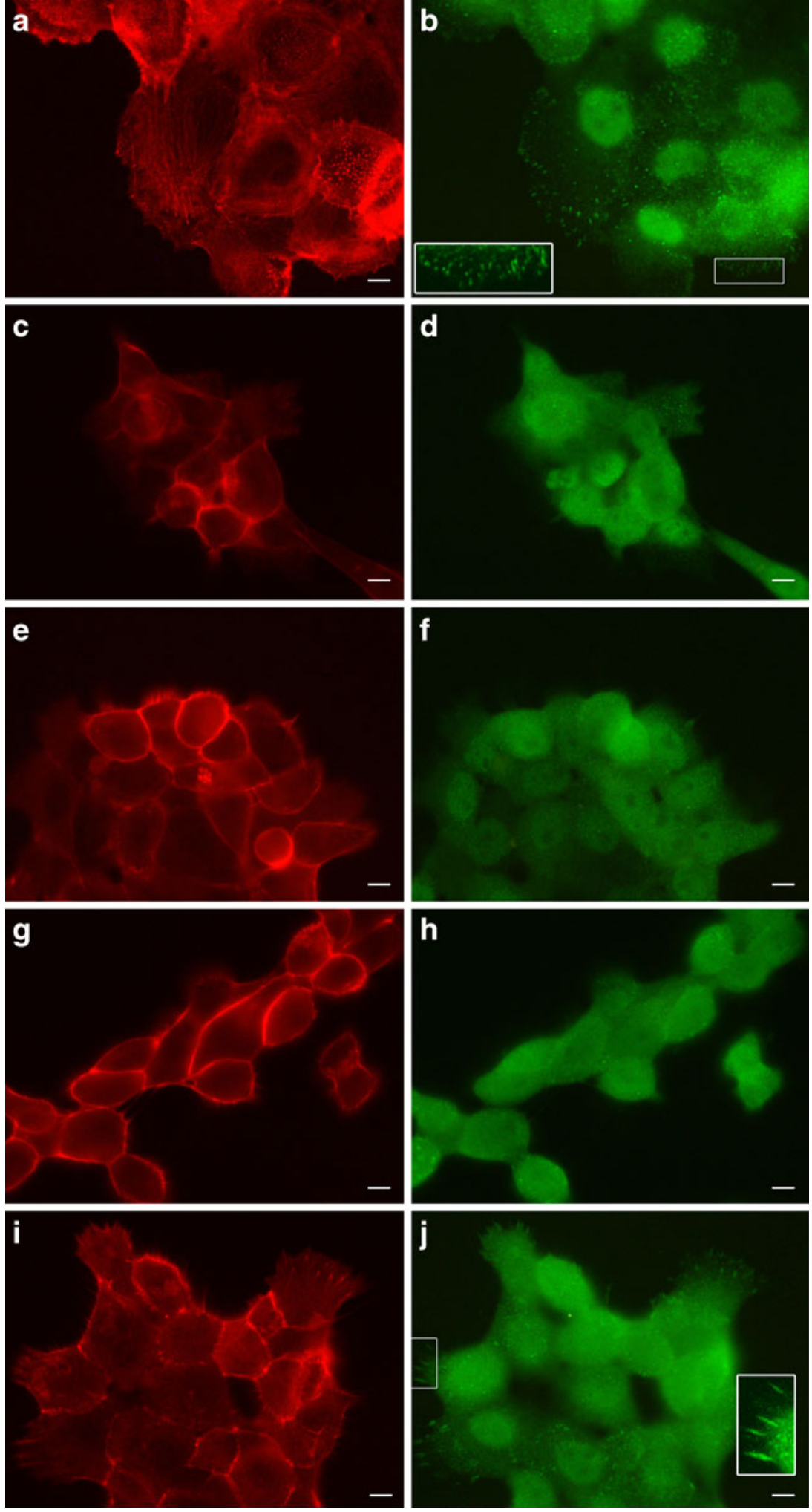

the centre of the magnet used. These settings result in the levitation of water, the major component of cells. Results from magnetic levitation were compared with data from cells exposed to a magnetic field of similar strengths, but without the gradient required for levitation, that is at $11.5 \mathrm{~T}$ in the centre of the magnetic field. This creates the possibility to discriminate between effects caused specifically by magnetic levita- 
tion or effects caused by the exposure of cells to the magnetic field. Finally, cells were also exposed to $0 \mathrm{~T}$ in the same experimental set up to control for any possible induced effects caused by other sources than the magnetic field or the magnetic levitation. Samples were evaluated using fluorescence microscopy and labelled for $\mathrm{F}$-actin and phospho-FAK ${ }^{397}$ in focal adhesions as described under Materials and Methods. PhosphoFAK $^{397}$ labelling was also observed in the nucleus of some cells. This is probably unspecific labelling and due to increased duration of fixation.

As shown in Fig. 4, levitation results in rounding of cells. Six minutes after the start of magnetic levitation a more pronounced cortical F-actin skeleton is visible,
Fig. 5 A431 cells exposed to a high magnetic field without levitation $(11.5 \mathrm{~T}$ in the center of the magnetic field) for $6 \min (\mathbf{a}, \mathbf{b}), 30 \mathrm{~min}(\mathbf{c}, \mathbf{d})$, $60 \mathrm{~min}(\mathbf{e}, \mathbf{f})$ and $120 \mathrm{~min}$ $(\mathbf{g}, \mathbf{h})$. Cells were fixed and labelled for F-actin (red) and phospho-FAK ${ }^{397}$ (green). Cells exposed to a magnetic field without levitation also responded with the rounding-up and subsequent spreading of cells. During rounding the cortical F-actin skeleton became more pronounced, retraction spikes became visible (arrow in

Fig. 4f) and phospho-FAK ${ }^{397}$ labelling in focal adhesions disappeared. After 120 min phospho-FAK ${ }^{397}$ labelling in focal adhesions reappeared again indicating the spreading of cells (h). Bar represents $10 \mu \mathrm{m}$, all inserts are enlarged twice
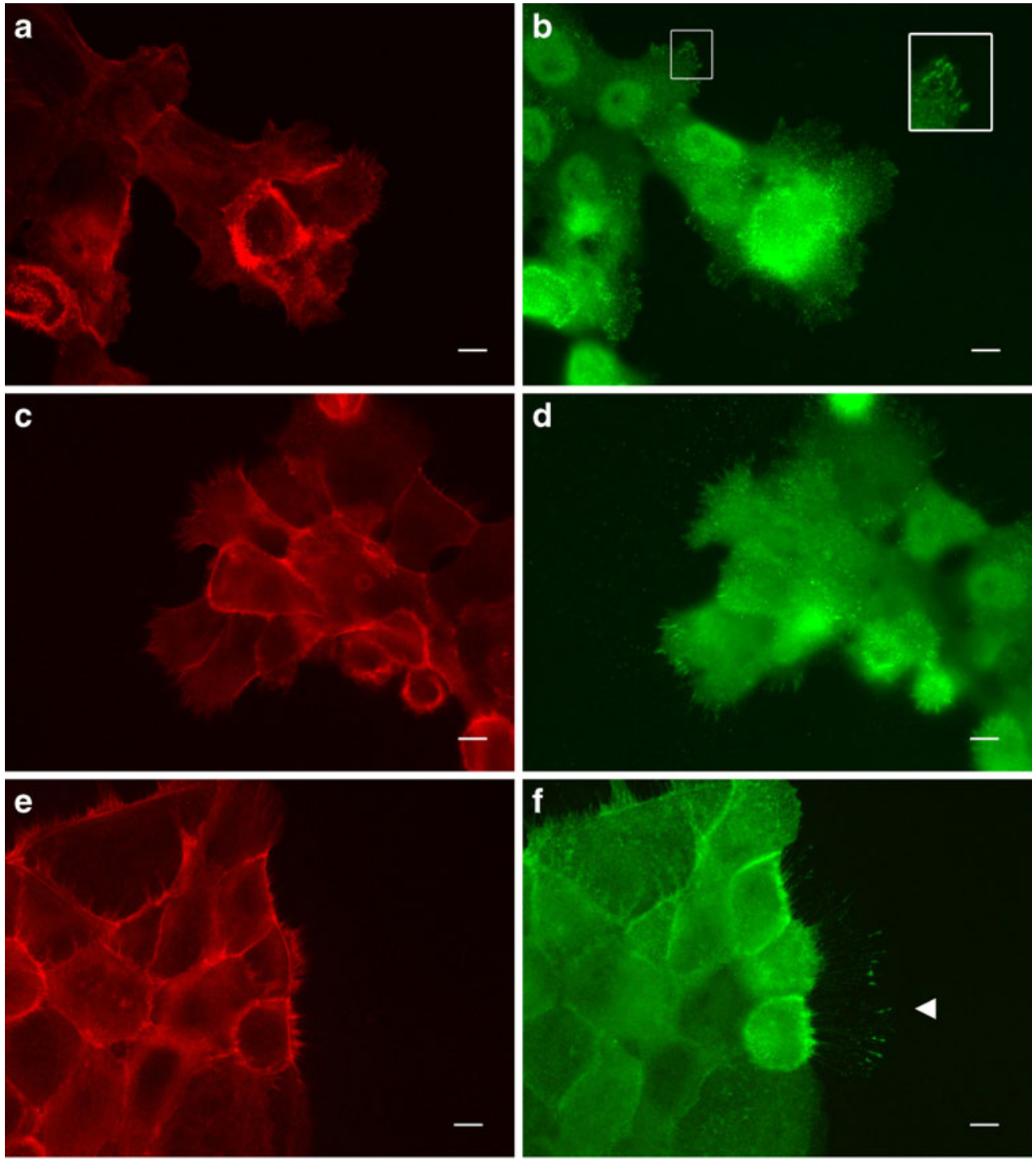

g

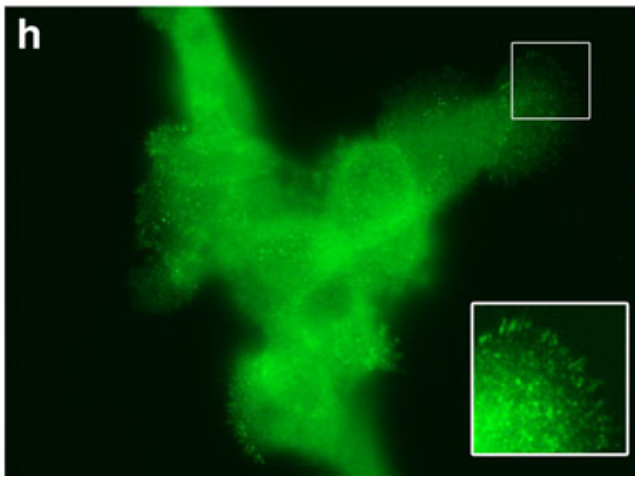


stress fibers (Fig. 4c) and phospho-FAK ${ }^{397}$ labelling in focal adhesions disappear (Fig. 4d) and the cells occupy less surface area. These effects become even more clear after 30 (Fig. 4e, f) and 60 min (Fig. 4g, h). In these samples the majority of cells show a rounded morphology. Starting from $60 \mathrm{~min}$ and continuing at $120 \mathrm{~min}$, cells start to spread and flatten again (Fig. 4i, j). New labelling of phospho-FAK ${ }^{397}$ in focal adhesions is visible (Fig. $4 \mathrm{j}$ ) and cells occupy a larger surface area. The number of stress fibers increases and the cortical F-actin skeleton becomes thinner (Fig. 4i).

\section{Exposure to a Magnetic Field of Identical Strength Compared to Magnetic Levitation}

During magnetic levitation cells are exposed to a gradient magnetic field. To control for effects caused by this magnetic field, cells were exposed to a field of identical strength without levitation, namely $11.5 \mathrm{~T}$ in the centre of the field. A similar pattern of rounding and spreading was observed as described for magnetic levitation (Fig. 4 vs. 5). After $6 \mathrm{~min}$ of exposure to the magnetic field, many peripheral focal adhesions were still stained for phospho-FAK ${ }^{397}$ (Fig. 5b). However, the number of labelled focal adhesions decreased after 30 and $60 \mathrm{~min}$ (Fig. 5d, f). Also retraction spikes became visible (arrow in Fig. 5f), indicating the rounding of cells. During the process of rounding the cortical actin skeleton became more pronounced and the number of stress fibers decreased (Fig. 5e). Both the level and rate of rounding of A431 cells seemed slightly less compared to cells exposed to magnetic levitation. This is indicated by the number of labelled focal adhesions present after 6 min of exposure (Fig. 4d vs. 5b) and also by the morphology of cells after $60 \mathrm{~min}$ of exposure (Fig. $4 \mathrm{~g}$ vs. $5 \mathrm{e}$ ). After $60 \mathrm{~min}$ of exposure to magnetic levitation, the cells are more rounded as compared to $60 \mathrm{~min}$ of exposure in the centre of the magnetic field indicated by the more pronounced cortical skeleton and absence of internal F-actin structures such as stress fibers. So the pattern of rounding and renewed spreading was comparable for both magnetic levitation and exposure to a field of identical strength.

To confirm that the described effects are indeed induced by the activated magnet and not by other sources, cells were also exposed to the same experimental set up without activation of the magnet $(0 \mathrm{~T})$. Control samples inside the magnet but without a magnetic field showed normal actin morphology and attachment as compared to regular lab controls (Fig. 6). Both after 30 min (Fig. 6a, b) and 60 min (Fig. 6c, d) cells exhibit abundant focal adhesions and showed a spread cell morphology. At these time points hardly any focal adhesions were observed in the samples that were subjected to the activated magnet (Figs. $4 \mathrm{e}-\mathrm{h}$ and $5 \mathrm{c}-\mathrm{f})$. Moreover, cells exposed to the activated magnet were clearly rounded at these time points in contrast to the cells that were subjected to identical treatment
Fig. 6 Control samples in the magnet without activation of the magnet $(0 \mathrm{~T})$. Cells were exposed to identical sheer stress, temperature profile and vibrations as compared to cells in the activated magnet, respectively during $30(\mathbf{a}, \mathbf{b})$ and $60 \mathrm{~min}(\mathbf{c}, \mathbf{d})$. Cells were fixed and labelled for F-actin (red) and phospho-FAK ${ }^{397}$ (green). Note the differences with the cells exposed to the activated magnet in Figs. $4 \mathrm{e}-\mathrm{h}$ and $5 \mathrm{c}-\mathrm{f}$
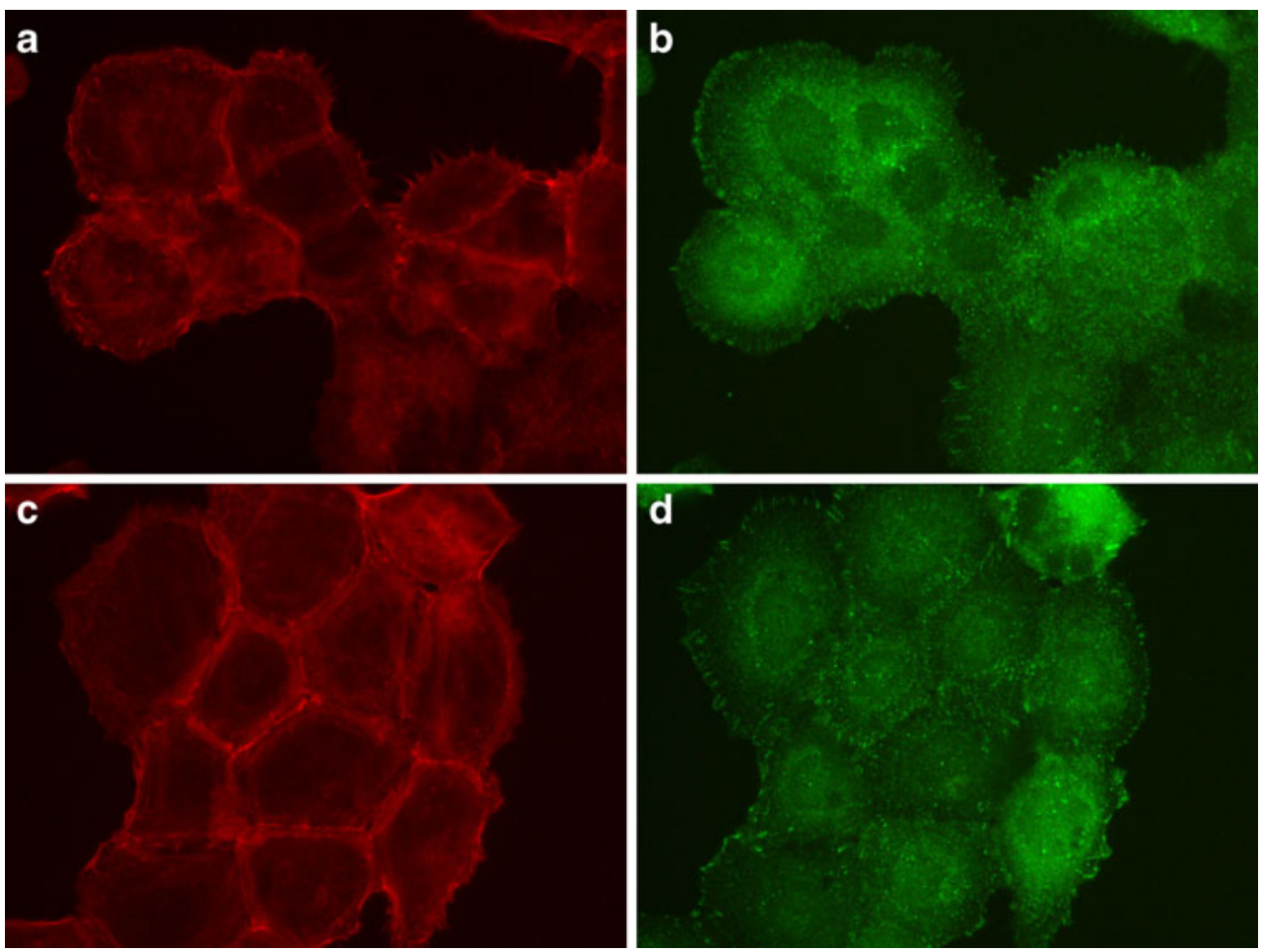
without activation of the magnet. This indicates that other factors like temperature profile, sheer stress due to medium refreshments or residual vibrations by the magnet cooling system in the experimental set up did not cause any additional effects. Therefore the described effects in Figs. 4 and 5 are caused by the magnetic field or the magnetic levitation.

\section{Discussion}

Experiments in space revealed gravity dependent cellular responses such as a changing gene expression, cytoskeletal changes and induction of apoptosis. Exposing cells to microgravity conditions in space provides insight in the sensitivity and responsiveness of cells to mechanical forces, such as gravity. However, access to perform experiments in space is limited and therefore there is a need for simulators allowing ground based simulated microgravity research. Two methods, rotation with the Random Positioning Machine and magnetic levitation, are used in this study to investigate induced morphological changes of human A431 cells. The focus was on the morphological changes of the F-actin cytoskeleton and its relation with attachment by focal adhesions. Both methods induced a transient process of cell rounding and subsequent reattachment and flattening of A431 cells. The results after 6 min are comparable to data obtained in real microgravity and in simulated microgravity using the fast rotating clinostat (Rijken et al. 1991a). This indicates that RPM rotation and magnetic levitation induce similar effects that are known to be caused by real microgravity.

Exposure A431 cells to 3D rotation in the RPM resulted in a transient process of rounding and subsequent reattachment and flattening of cells. Rounded cells were visible after 6 min of rotation illustrated by an abundant actin cortical skeleton and the disappearance of actin stress fibers. This was also observed in real microgravity during sounding rocket missions and in simulated microgravity using the fast rotating clinostat (Rijken et al. 1991a). The rounding of cells was further illustrated by the disappearance of focal adhesions. After this rounding up of cells, the cells adapt to their new environment and reattach and flatten. This is illustrated by the reappearance of focal adhesions, the cells occupy more surface, stress fibers reappear and the cortical skeleton becomes less abundant.

The Random Positioning Machine (Hoson et al. 1992, 1997; van Loon 2007; Borst and van Loon 2009) is based on the principle that the direction of gravity is randomized in three dimensions. Rotation in three dimensions with varying speeds is required to provide truly three dimensional random motion (Borst and van Loon 2009). This variation in velocity might result in a complicated pattern of induced effects in cells, since the speed of rotation was described to be an important variable in the fast rotating clinostat that ideally rotates in one dimension (Briegleb 1992).

Increasing the speed of rotation will reduce residual gravity perception, but will increase centrifugal forces (Briegleb 1992). The perception of gravity might be dependent on the process under investigation and reflects the time lag phase of the gravity sensitive element of the process under investigation. This implies that the speed of rotation should be optimized for each different process studied, avoiding both residual levels of gravity perception and perception of centrifugal forces. For A431 cells exposed to two dimensional rotation, both the level and the speed of cell rounding and renewed spreading indeed varied with the selected speed of rotation (data not shown).

Recently, the RPM is used as a simulator for microgravity in various studies investigating responses in animal cells (for example Grimm et al. 2002; Uva et al. 2002; Infanger et al. 2006; Pardo et al. 2005; Patel et al. 2009; Meloni et al. 2006). However, the mode of operation of the RPM that was used in these studies varies. Both 10 and $60 \mathrm{rpm}$ are used frequently, sometimes random speed was used and often the mode of operation and the location of the sample within the system are not mentioned. Since for the fast rotating clinostat the mode of operation, specifically the speed of rotation, was related to variation in cellular responses (Briegleb 1992; own unpublished data), it is critical to clarify the chosen mode of operation for the RPM more precise. In this study the microgravity target that was selected was the actin cytoskeleton and its relation to attachment via focal adhesions. Other microgravity sensitive components in cells might possess different sensitivity and responsiveness towards their mechanical environment, such as gravity. Therefore, it is important to validate the mode of rotation for each experiment involving simulation of microgravity by the RPM.

Another limitation of simulation by randomization of the direction of gravity in the RPM is that the randomization requires time to statistically nullify gravity in all directions. Therefore only processes can be studied that experience a certain time lag phase. For example fast occurring processes might not be suitable for studying in the RPM. So, on the one hand the specific microgravity sensitive target in the cell requires a specific mode of operation of the RPM to simulate microgravity. On the other hand there are limitations in the applicability of the RPM that are associated with its design and mode of operation. More awareness of 
the importance and consequences of the selection of the mode of operation of the RPM is required. The selection of a certain mode of operation and maximum speed is no guarantee for simulating real microgravity. Moreover, the physical characterization of the RPM, such as fluid dynamics, should be investigated in more detail (van Loon 2007) as was done for the fast rotating clinostat (Briegleb 1992; Albrecht-Buehler 1992). Most importantly, results measured under conditions of simulated microgravity should be confirmed in real microgravity.

Interestingly, exposing A431 cells to magnetic levitation resulted in a similar pattern of rounding followed by spreading as was observed in the case of RPM rotation. The responses that were observed after $6 \mathrm{~min}$ of levitation were very similar to responses observed in cells exposed during 6 min to real microgravity using sounding rockets. This indicates that magnetic levitation induces effects that are known to be caused by microgravity.

However, exposing cells to a magnetic field of identical strength but without the levitating gradient resulted in similar responses. This raises concerns about the relevance of the data obtained with magnetic levitation experiments. For the rounding and spreading of A431 cells no clear discrimination was possible between effects caused by the high magnetic field itself and by magnetic simulation of microgravity using a gradient magnetic field of identical strength.

The use of high gradient magnetic fields allows the simulation of microgravity almost instantly and allows one to study fast occurring biological processes in contrast to other existing simulators, such as the clinostat and RPM where more time might be required to establish an averaged gravity vector randomization. For this reason magnetic levitation is an interesting additional device for simulating microgravity conditions.

The responses of cells exposed to high magnetic fields give rise to concerns about the side effects introduced by magnetic levitation. The rounding of A431 cells caused by exposure to a high gradient magnetic field without levitation appears undistinguishable from the reported rounding of A431 cells in real microgravity (Rijken et al. 1991a). This indicates the importance of this control when performing magnetic levitation studies. Studies claiming the usefulness of magnetic levitation in simulating microgravity might easily draw wrong conclusions when not performing such controls at the same field strength.

Other studies also reported responses in cells exposed to magnetic fields (Denegre et al. 1998; Valiron et al. 2005). Also the influence of high magnetic fields on the behaviour of actin was reported, both in vitro
(Torbet and Dickens 1984) and in vivo (Valiron et al. 2005). This effect is most probably caused by magnetic orientation of actin. Long biomolecules, like actin and DNA, exhibit an anisotropic magnetic susceptibility that tends to align them in a magnetic field (Torbet and Dickens 1984; Maret and Dransfeld 1985; Christianen et al. 2004).This suggests that side effects are unavoidable when using magnetic levitation as a simulator for microgravity. However, when there is a clear additional microgravity sensitive response in cells exposed to magnetic levitation, it might still be possible to recognize specific cellular responses to simulated microgravity by such studies.

For the described pattern of rounding and spreading of A431 cells, the discrimination between responses to simulated microgravity and exposure to a high magnetic field seems not possible. Therefore, it will be interesting to study other cell lines and cellular responses under conditions of magnetic simulation of microgravity.

In conclusion, RPM rotation and magnetic levitation of A431 cells resulted in a very similar response of cell rounding and subsequent flattening. The induction of cell rounding is known from experiments in real microgravity. However, both methods also have disadvantages that can easily lead to misinterpretation of results. Therefore, it is required to confirm that responses are induced by the simulation of microgravity and not caused by side effects. The further characterization of both methods is required and most importantly, results measured under conditions of simulated microgravity should be confirmed in real microgravity.

Acknowledgements This work is subsidized by the Dutch Space Organization (SRON, grant MG-057 (van Loon) and MG-059 (Moes)). Part of this work has also been supported by EuroMagNET under EU contract RII3-CT-2004-506239 and the "Stichting voor Fundamenteel Onderzoek der Materie (FOM)", financially supported by the "Nederlandse Organisatie voor Wetenschappelijk Onderzoek (NWO)".

Open Access This article is distributed under the terms of the Creative Commons Attribution Noncommercial License which permits any noncommercial use, distribution, and reproduction in any medium, provided the original author(s) and source are credited.

\section{References}

Albrecht-Buehler, G.: The simulation of microgravity conditions on the ground. ASGSB Bull. 5, 3-10 (1992)

Beaugnon, E., Tournier, R.: Levitation of organic materials. Nature 349, 470-470 (1991a) 
Beaugnon, E., Tournier, R.: Levitation of water and organic substances in high static magnetic fields. J. Phys. III France 1, 1423-1428 (1991b)

Berry, M.V., Geim, A.K.: Of flying frogs and levitrons. Eur. J. Phys. 18, 307-313 (1997)

Boonstra, J.: Growth factor-induced signal transduction in adherent mammalian cells is sensitive to gravity. FASEB J. 13, S35-S42 (1999)

Boonstra, J., Moes, M.J.: Signal transduction and actin in the regulation of G1-phase progression. Crit. Rev. Eukaryot. Gene Expr. 15, 255-276 (2005)

Borst, A.G., van Loon, J.J.W.A.: Technology and developments for the random positioning machine, RPM. Microgravity Sci. Technol. 21, 287-292 (2009)

Briegleb, W.: Some quantitative aspects of the fast-rotating clinostat as a research tool. ASGSB Bull. 5, 23 (1992)

Carmeliet, G., Vico, L., Bouillon, R.: Space flight: a challenge for normal bone homeostasis. Crit. Rev. Eukaryot. Gene Expr. 11, 131-144 (2001)

Christianen, P.C.M., Shklyrevskiy, I.O., Boamfa, M.I., Maan, J.C.: Alignment of molecular materials in high magnetic fields. Physica B 346-347, 255-261 (2004)

de Groot, R.P., Rijken, P.J., den Hertog, J., Boonstra, J., Verkleij, A.J., de Laat, S.W., Kruijer, W.: Microgravity decreases c-fos induction and serum response element activity. J. Cell Sci. 97, 33-38 (1990)

de Groot, R.P., Rijken, P.J., Boonstra, J., Verkleij, A.J., de Laat, S.W., Kruijer, W.: Epidermal growth factor-induced expression of c-fos is influenced by altered gravity conditions. Aviat. Space Environ. Med. 62, 37-40 (1991a)

de Groot, R.P., Rijken, P.J., den Hertog, J., Boonstra, J., Verkleij, A.J., de Laat, S.W., Kruijer, W.: Nuclear responses to protein kinase $\mathrm{C}$ signal transduction are sensitive to gravity changes. Exp. Cell Res. 197, 87-90 (1991b)

Denegre, J.M., Valles, J.M. Jr, Lin, K., Jordan, W.B., Mowry, K.L.: Cleavage planes in frog eggs are altered by strong magnetic fields. Proc. Natl. Acad. Sci. U.S.A. 95, 14729-14732 (1998)

dos Remedios, C.G., Chhabra, D., Kekic, M., Dedova, I.V., Tsubakihara, M., Berry, D.A., Nosworthy, N.J.: Actin binding proteins: regulation of cytoskeletal microfilaments. Physiol. Rev. 83, 433-473 (2003)

Fitts, R.H., Riley, D.R., Widrick, J.J.: Functional and structural adaptations of skeletal muscle to microgravity. J. Exp. Biol. 204, 3201-3208 (2001)

Fritsch-Yelle, J.M., Charles, J.B., Jones, M.M., Wood, M.L.: Microgravity decreases heart rate and arterial pressure in humans. J. Appl. Physiol. 80, 910-914 (1996)

Geim, A.: Everyone's magnetism. Phys. Today 51, 36-39 (1998)

Genersch, E., Schneider, D.W., Sauer, G., Khazaie, K., Schuppan, D., Lichtner, R.B.: Prevention of EGF-modulated adhesion of tumor cells to matrix proteins by specific EGF receptor inhibition. Int. J. Cancer 75, 205-209 (1998)

Glade, N., Beaugnon, E., Tabony, J.: Ground-based methods reproduce space-flight experiments and show that weak vibrations trigger microtubule self-organisation. Biophys. Chemist. 121, 1-6 (2006)

Grimm, D., Bauer, J., Kossmehl, P., Shakibaei, M., Schöberger, J., Pickenhahn, H., Schulze-Tanzil, G., Vetter, R., Eilles, C., Paul, M., Cogoli, A.: Simulated microgravity alters differentiation and increases apoptosis in human follicular thyroid carcinoma cells. FASEB J. 16, 604-606 (2002)

Hammond, T.G., Lewis, F.C., Goodwin, T.J., Linnehan, R.M., Wolf, D.A., Hire, K.P., Campbell, W.C., Benes, E., O'Reilly, K.C., Globus, R.K., Kaysen, J.H.: Gene expression in space. Nat. Med. 5, 359 (1999)
Heijna, M.C.R., Poodt, P.W.G., Tsukamoto, K., de Grip, W.J., Christianen, P.C.M., Maan, J.C., Hendrix, J.L.A., van Enckevort, W.J.P., Vlieg, E.: Magnetically controlled gravity for protein crystal growth. Appl. Phys. Lett. 90, 264105 (2007)

Hoson, T., Kamisaka, S., Masuda, Y., Yamashita, M.: Changes in plant growth processes under microgravity conditions simulated by a three-dimensional clinostat. Bot. Mag. 105, 53-70 (1992)

Hoson, T., Kamisaka, S., Masuda, Y., Yamashita, M., Buchen, B.: Evaluation of the three-dimensional clinostat as a simulator of weightlessness. Planta 203, S187-S197 (1997)

Hughes-Fulford, M.: Function of the cytoskeleton in gravisensing during spaceflight. Adv. Space Res. 32, 1585-1593 (2003)

Infanger, M., Kossmehl, P., Shakibaei, M., Bauer, J., KossmehlZorn, S., Cogoli, A., Curcio, F., Oksche, A., Wehland, M., Kreutz, R., Paul, M., Grimm, D.: Simulated weightlessness changes the cytoskeleton and extracellular matrix proteins in papillary thyroid carcinoma cells. Cell Tissue Res. 324, 267 277 (2006)

Liu, Y., Wang, E.: Transcriptional analysis of normal human fibroblast responses to microgravity stress. Genom. Proteom. Bioinform. 6, 29-41 (2008)

Maret, G., Dransfeld, K.: Biomolecules and Polymers in High Steady Magnetic Fields in Strong and Ultrastrong Magnetic Fields and their Applications, Chapter 4. Springer, Berlin (1985)

Meloni, M.A., Galleri, G., Pippia, P., Cogoli-Greuter, M.: Cytoskeleton changes and impaired motility of monocytes at modelled low gravity. Protoplasma 229, 243-249 (2006)

Nelson, J.M., Fry, D.W.: Cytoskeletal and morphological changes associated with the specific suppression of the epidermal growth factor receptor tyrosine kinase activity in A431 human epidermoid carcinoma. Exp. Cell Res. 233, 383-390 (1997)

Papaseit, C., Pochon, N., Tabony, J.: Microtubule self-organization is gravity-dependent. Proc. Natl. Acad. Sci. U.S.A. 97, 8364-8368 (2000)

Pardo, S.J., Patel, M.J., Sykes, M.C., Platt, M.O., Boyd, N.L., Sorescu, G.P., Xu, M., van Loon, J.J., Wang, M.D., Jo, H.: Simulated microgravity using the Random Positioning Machine inhibits differentiation and alters gene expression profiles of 2T3 preosteoblasts. Am. J. Physiol., Cell Physiol. 288, C1211-C1221 (2005)

Patel, M.J., Chang, K.H., Sykes, M.C., Talish, R., Rubin, C., Jo, H.: Low magnitude and high frequency mechanical loading prevents decreased bone formation responses of $2 \mathrm{~T} 3$ preosteoblasts. J. Cell. Biochem. 106, 306-316 (2009)

Perenboom, J.A.A.J., Wiegers, S.A.J., Christianen, P.C.M., Zeitler, U., Maan, J.C.: Research in high magnetic fields: the installation at the university of Nijmegen. J. Low Temp. Phys. 133, 181-201 (2003)

Rijken, P.J., de Groot, R.P., Briegleb, W., Kruijer, W., Verkleij, A.J., Boonstra, J., de Laat, S.W.: Epidermal growth factorinduced cell rounding is sensitive to simulated microgravity. Aviat. Space Environ. Med. 62, 32-36 (1991a)

Rijken, P.J., Hage, W.J., van Bergen en Henegouwen, P.M., Verkleij, A.J., Boonstra, J.: Epidermal growth factor induces rapid reorganization of the actin microfilament system in human A431 cells. J. Cell Sci. 100, 491-499 (1991b)

Schenck, J.F.: Health and physiological effects of human exposure to whole-body four-tesla magnetic fields during MRI. Ann. Rev. Acad. Sci. 649, 285 (1992)

Schwarzenberg, M., Pippia, P., Meloni, M.A., Cossu, G., Cogoli-Greuter, M., Cogoli, A.: Microgravity simulations 
with human lymphocytes in the free fall machine and in the random positioning machine. J. Gravit. Physiol. 5, P23-P26 (1998)

Tabony, J., Rigotti, N., Glade, N., Cortès, S.: Effect of weightlessness on colloidal particle transport and segregation in selforganising microtubule preparations. Biophys. Chem. 127, 172-180 (2007)

Torbet, J., Dickens, M.J.: Orientation of skeletal muscle actin in strong magnetic fields. FEBS Lett. 173, 403-406 (1984)

Ulbrich, C., Westphal, K., Baatout, S., Wehland, M., Bauer, J., Flick, B., Infanger, M., Kreutz, R., Vadrucci, S., Egli, M., Cogoli, A., Derradji, H., Pietsch, J., Paul, M., Grimm, D.: Effects of basic fibroblast growth factor on endothelial cells under conditions of simulated microgravity. J. Cell. Biochem. 104, 1324-1341 (2008)

Ullrich, O., Huber, K., Lang, K.: Signal transduction in cells of the immune system in microgravity. Cell. Commun. Signal. 6, 9 (2008)

Uva, B.M., Masini, M.A., Sturla, M., Prato, P., Passalacqua, M., Giuliani, M., Tagliafierro, G., Strollo, F.: Clinorotation- induced weightlessness influences the cytoskeleton of glial cells in culture. Brain Res. 934, 132-139 (2002)

Valiron, O., Peris, L., Rikken, G., Schweitzer, A., Saoudi, Y., Remy, C., Job, D.: Cellular disorders induced by high magnetic fields. J. Magn. Reson. Imaging 22, 334-340 (2005)

Valles, J.M. Jr, Lin, K., Denegre, J.M., Mowry, K.L.: Stable magnetic field gradient levitation of Xenopus laevis: toward low-gravity simulation. Biophys. J. 73, 1130-1133 (1997)

van Loon, J.J.W.A.: Some history and use of the random positioning machine, RPM, in gravity related research. Adv. Space Res. 39, 1161-1165 (2007)

Versari, S., Villa, A., Helder, M.N., Doulabi, B.Z., van Loon, J., Bradamante, S.: Effects of gravity on proliferation and differentiation of adipose tissue-derived stem cells. J. Gravit. Physiol. 14, 127-128 (2007a)

Versari, S., Villa, A., Bradamante, S., Maier, J.A.M.: Alterations of the actin cytoskeleton and increased nitric oxide synthesis are common features in human primary endothelial cell response to changes in gravity. Biochim. Biophys. Acta 1773, $1645-1652$ (2007b) 